\title{
Mass measurements and ion-manipulation techniques applied to the heaviest elements
}

\author{
Michael Block $\mathrm{B}^{1,2,3, \mathrm{a}}$ \\ ${ }^{1}$ GSI Helmholtzzentrum für Schwerionenforschung, 64291 Darmstadt, Germany \\ ${ }^{2}$ Helmholtz Institute Mainz, 55099 Mainz, Germany \\ ${ }^{3}$ Johannes Gutenberg-Universität, 55099 Mainz, Germany
}

\begin{abstract}
High-precision mass measurements of radionuclides with stateof-the-art mass spectrometry allows us to obtain accurate binding energies. These reflect changes in the nuclear structure. Two-nucleon separation energies, for example, are sensitive indicators of shell closures and the onset of deformation. In addition, masses provide a benchmark for theoretical nuclear models and support their improvement with respect to a better predictive power. In the region of the heaviest elements this enables mapping the strength of shell effects and their extension as recently demonstrated for $N=152$. Accurate masses can furthermore provide anchor points to fix decay chains in the mass surface. Moreover, advanced ion-manipulation techniques developed in the context of mass spectrometry pave the way for novel types of experiments such as trap-assisted decay spectroscopy of nuclear state-selected samples. This opens new perspectives for a mass number assignment of decay chains originating from yet unknown superheavy nuclides.
\end{abstract}

\section{Introduction}

Ion-trap based techniques allow us to manipulate ions at low energy in a well-controlled manner to prepare pure samples of radioactive ions for precision measurements. One of the most prominent applications for radioactive nuclides is Penning trap mass spectrometry (PTMS) $[1,2]$. In this approach, the mass of an ion is obtained via a measurement of the cyclotron frequency $v_{c}=q \mathrm{~B} / \mathrm{m}$ at which the ion orbits in the confining electromagnetic fields of a Penning trap. The typical relative precision that is nowadays obtained for the masses of radionuclides is on the order of $10^{-8}$ for short-lived nuclides [3]. For long-lived and stable particles even two to three orders of magnitude lower uncertainties have been reached $[4,5]$. The ingredients for mass measurements with highest precision are the selective removal of unwanted nuclides and the minimization of systematic effects well below the anticipated uncertainty. The most common systematic effects in PTMS include the spatial and temporal homogeneity of the confining fields and the ion-ion Coulomb interaction. The systematic effects in PTMS and their impact on the mass uncertainty is well understood and has been discussed in the literature in depth $[6,7]$. In general, the measurements in a oneion-at-a-time procedure and the application of appropriate ion cooling methods reduces the amplitudes of the ion motion such that the ions only probe the most homogeneous region of the trapping fields. Confinement in an ultra-high vacuum environment furthermore reduces

\footnotetext{
a e-mail: m.block@gsi.de
} 
collisions with rest gas atoms. Using appropriate calibrating ions, moreover, absolute mass measurements are feasible using carbon cluster ions as mass reference, for example.

In the context of nuclear physics, and in particular for investigation of the nuclear properties of the heaviest elements, two main features of mass measurements are of interest. On the one hand side the nuclear structure evolution can be tracked by masses and mass differences to identify, for example, shell closures and the onset of deformation. Moreover, accurate direct mass measurements provide anchor points in the mass surface to pin down decay chains originating from superheavy nuclides as demonstrated for example for nobelium isotopes [8]. On the other hand, the high mass resolving power provided by Penning trap techniques is suitable for the separation of unwanted by-products to prepare pure radioactive samples for subsequent experiments such as nuclear decay spectroscopy. These so-called trap-assisted decay spectroscopy experiments have already been performed in lighter nuclides [9] demonstrating that even nuclear state-selected samples can be delivered to a decay spectroscopy setup for certain nuclides [10]. Trap-assisted decay spectroscopy of isotopeselected samples opens new perspectives for an $A / Q$ identification of superheavy nuclides, an application of particular interest for nuclides originating from yet unknown elements.

\section{Mass measurements with Penning traps}

PTMS has been a well-established mature field for many years [2]. However, the extension to the heaviest nuclides was only achieved less than ten years ago with pioneering measurement with SHIPTRAP at GSI Darmstadt [11]. The major challenges that had to be mastered are the rather low yield and the high energy of the nuclides of interest following their production in nuclear fusion reactions. The present status of PTMS of the heaviest elements has been summarized in recent review articles $[12,13]$. Here, the essence of the method with respect to its application to the heaviest elements will be briefly summarized in the following.

The radionuclides of interest are produced in complete fusion-evaporation reactions. Following the separation from the primary beam in an electromagnetic recoil separator, the reaction products are provided with energies in the order of some ten $\mathrm{MeV}$ for experiments. So-called buffer gas stopping cells are utilized, also in the case of SHIPTRAP [14], to convert these energetic ions of interest into a low-energy beam suitable for ion trapping. These buffer gas stopping cells, sometimes also referred to as ion catchers, slow down the reaction products in an inert buffer gas atmosphere, usually helium, at pressures of about 50-100 mbar in some ten centimeters. Depending on the element and its ionization potential the major fraction of the slowed ions remain singly- or doubly-charged. These can be extracted within tens of milliseconds from the buffer gas by a combination of electric fields and gas flow through an extraction nozzle. First-generation stopping cells have reached overall efficiencies of about $5-10 \%$ [14, 15]. Recently, first cryogenic devices have been introduced boosting the efficiency to a level of about $30-50 \%$ [16, 17].

The extracted ions are then transported, cooled, and bunched by radio-frequency quadrupole ion guides. The formed low-energy bunched ion beam is injected into a Penning trap system where the mass of the ion is measured. Pioneering experiments on nobelium and lawrencium nuclides around the deformed neutron shell closure at $N=152$ have been performed with the Penning trap system SHIPTRAP [11, 18]. It consists of two cylindrical Penning traps that are installed in the bore of a superconducting solenoid featuring two homogeneous centers. The first trap is dedicated to ion preparation by a mass-selective buffer gas cooling technique that allows removing unwanted by-products that may accompany the ions of interest. The mass resolving power achieved in this step is on the order of 100000 , sufficient to separate nuclear isobars. This is, however, not an issue for the heaviest elements 
where the ions of interest are typically produced in $x n$-evaporation channels of complete fusion reactions. In the second trap mass measurements can be performed using different techniques. The most exotic nuclides such as ${ }^{256} \operatorname{Lr}[18]$ have been measured by the socalled time-of-flight ion-cyclotron-resonance (ToF-ICR) method as described in reference [19]. This method is rather robust and universal and can be applied to radionuclides with half-lives exceeding about $10 \mathrm{~ms}$. The achievable precision scales inversely proportional with the excitation (and hence measurement) time. The main drawback of this method is the need to scan the excitation frequency around the expected cyclotron frequency and the corresponding number of 30-50 ions that needs to be accumulated in order to determine a mass value. In the above mentioned case of ${ }^{256} \mathrm{Lr}$ a single measurement took about four days, which is challenging as many parameters have to be kept constant to achieve a high precision. This measurement time can be further reduced with a number of upgrades some of which are addressed in Sect. 4. Ultimately single-ion mass measurement techniques have to be employed to foray into the territory of the heaviest elements.

Mass spectrometers such as Penning traps with their high mass resolving power are also useful isobar separators. In many cases even isomeric states can be separated. This opens up interesting perspectives for novel types of experiments. Trap-assisted decay spectroscopy is a powerful combination of Penning traps or other mass separators with decay spectroscopy setups. In the region of heavy nuclides that predominantly decay by $\alpha$ decay efficient and compact $\alpha-\gamma$ detection setups are suitable. The feasibility of this approach has been demonstrated combing TASISpec [20] with SHIPTRAP [21], for example. Purified and potentially state-selected tailored samples were implanted into the setup where the decay is measured. Similar experiments have already been performed for $\beta$-decay studies, for example in Jyväskylä [9] and specifically for measurements of superallowed $\beta$ decays. The feasibility of $\alpha-\gamma$ spectroscopy was demonstrated for Rn and Ra isotopes in proof-ofprinciple experiments connecting TASISpec and SHIPTRAP. Due to the purity of the samples this approach is particularly useful for measuring branching ratios of weak decay branches accurately. This has been shown for example for ${ }^{213} \mathrm{Ra}$ [21].

Another opportunity for applying this technique is the mass identification of members of decay chains originating from yet unknown superheavy nuclides. To this end only a moderate mass resolving power on the order of 300 suffices to distinguish different isotopes. Such a mass resolving power can already be realized with radio frequency mass filters, for example, but of course also with Penning traps and time-of-flight mass spectrometers. Since the slowing down, cooling and bunching of the ions of interest takes place on a time scale on the order of 10-100 milliseconds, this method can be applied to relatively short-lived nuclides. Alternative ideas for mass-selected decay spectroscopy for the $A / Q$ identification of superheavy nuclides are pursued within the FIONA project at Berkeley [22]. There an electromagnetic separator, a Wien filter with unbalanced electric and magnetic field will be utilized. Mass spectrometric $A / Q$ identification techniques with the high resolution achieved in ion traps also provide an option for the detection of long-lived nuclides and nuclides that do not exhibit $\alpha$ decay or spontaneous fission, if a competitive overall efficiency is achieved.

\section{Mass measurements and nuclear structure}

A key question with respect to the nuclear structure evolution in the heaviest elements is related to the character of shell effects. While in lighter nuclei, for example in the tin isotopic chain, well localized and strong shell closures are observed, in the heaviest nuclides a different behaviour is predicted, see for example [23]. Different nuclear models obtain rather extended regions of enhanced shell stabilization in contrast to well-localized magic numbers. 


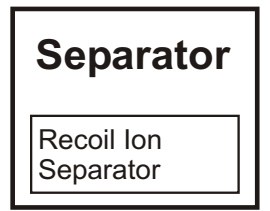

Production and Separation

Tens of $\mathrm{MeV}$

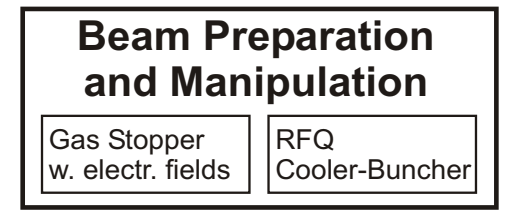

Slowing down Cooling and and Extraction bunching

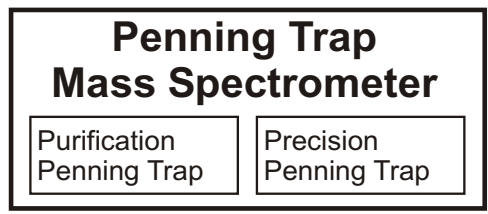

Purification and preparation

High-Precision

Measurement

Some eV

Figure 1. Schematic layout of a Penning trap mass spectrometer suitable for the heaviest elements.

In addition, the emergence of deformed shell closures in the regions of neutron numbers $N=152$ and $N=162$ have been predicted and experimentally observed, originally based on experimental $Q_{\alpha}$ values. The present experimental situation is illustrated by the two-neutron separation energies for the elements from $Z=98-100$ as displayed in Fig. 1. The values have been calculated based on the experimental data compiled in the most recent Atomic Mass Evaluation (AME 2013) [24]. Despite the large uncertainties in the more exotic nuclides indications of shell closures at $N=152$ and $N=162$ are clearly visible.

The AME 2013 considered the results of direct mass measurements performed with SHIPTRAP for the accurate mapping of the $N=152$ shell effects in nobelium and lawrencium $[11,18]$. This deformed shell closure was found to be about $\delta_{2 n}=1.2 \mathrm{MeV}$. The $N=152$ shell is most pronounced at $Z=100$ with a shell gap of about $\delta_{2 n}=1.5 \mathrm{MeV}$. The evolution for different proton numbers is yet not fully established due to a lack of experimental data with sufficient precision. In principle, direct mass measurements are an ideal tool for such studies. However, the low yield of the nuclides of interest for such measurements makes this endeavor tedious. In lighter nuclides between uranium and fermium the availability of macroscopic amounts for certain long-lived nuclides facilitates off-line measurements of some relevant nuclides. First measurements have been performed for the masses of Am, Cm, and Cf nuclides with TRIGA-TRAP [25] and will be extended in the near future. A local mass evaluation in the spirit of the the Atomic Mass Evaluation (AME) furthermore indicated that already a few accurate direct mass measurements can impact the masses of many more nuclides in the region via numerous established decay links.

Thus, a promising strategy for the future is to combine mass spectrometry and $\alpha-\gamma$ decay spectroscopy: direct mass measurements provide the masses of selected anchor points with high accuracy, which is in particular relevant for odd-odd and odd- $A$ nuclides. In these nuclides the strongest $\alpha$-decay branch usually populates excited states. Then, the accurate determination of the mass values from a $Q_{\alpha}$ value require a detailed knowledge of the decay scheme. For even-even nuclides the situation is rather straightforward as the strongest $\alpha$ transitions typically connects the ground states in the mother and daughter nuclides. The different data can be fed into the network of the AME where the masses of all nuclides are evaluated based on known data and their links. The impact of even a few accurate data has been demonstrated for example for the masses of the nobelium isotopes measured at SHIPTRAP [8] and for masses of actinides measured at TRIGA-TRAP [25].

While the experimental data around $N=152$ is already rather comprehensive the situation for $N=162$ is worse as indicated by the large uncertainties of many masses in Fig. 2. In addition, the masses of certain nuclides are only known from extrapolations. Nonetheless, the shell effects at $N=162$ are clearly visible. For many of these nuclides direct mass measurements are presently out of reach due to their low yield and rather short half-lives for some neutron-deficient nuclides. However, even for detailed $\alpha-\gamma$ spectroscopy 


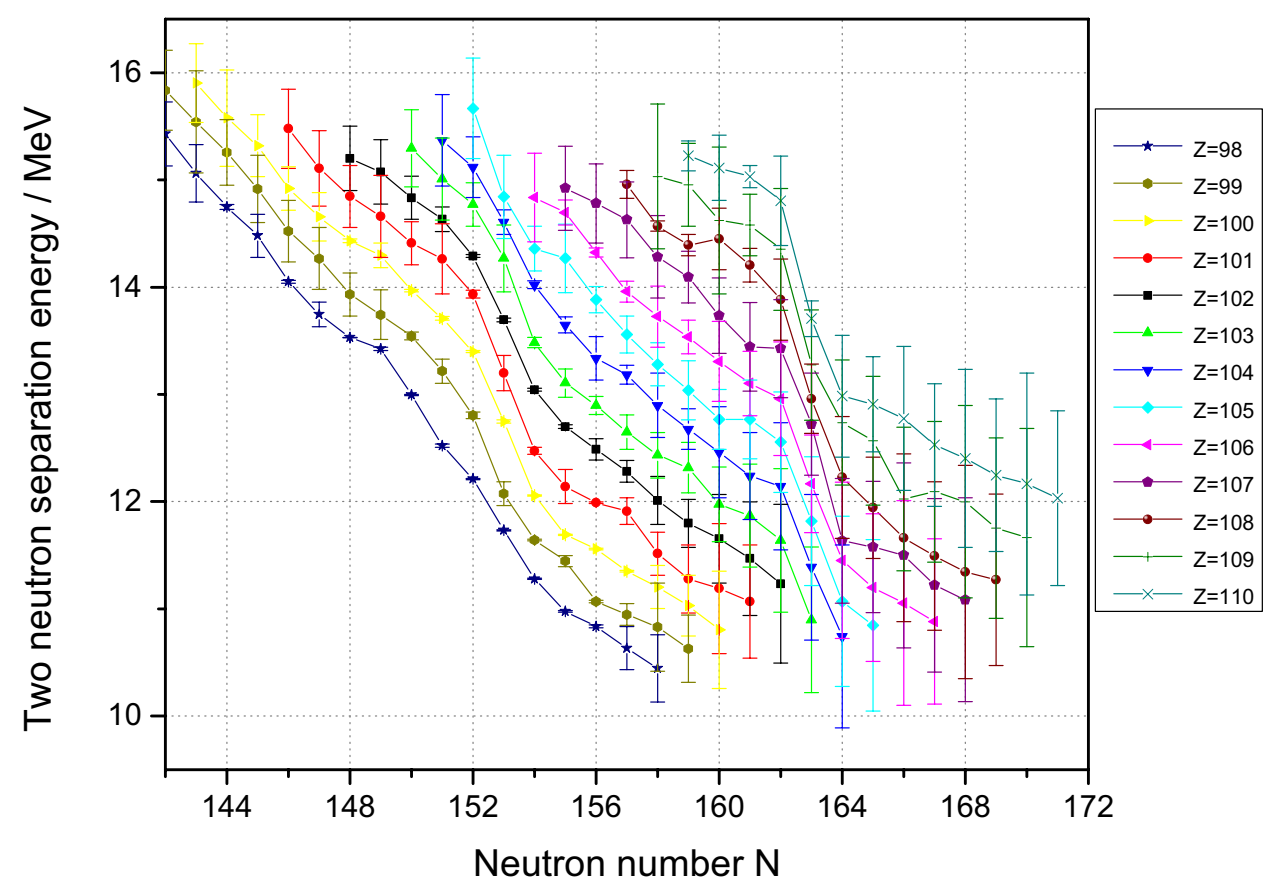

Figure 2. Two neutron separation energies for the elements from $Z=98-100$. The data are calculated based on the masses from reference [24].

the low cross sections, for example a few picobarn for the Ds isotopes ${ }^{268-270}$ Ds, are also challenging.

\section{Future perspectives for mass measurements of heavy elements}

At present a mass measurement program for nuclides in the region above fermium is ongoing using the SHIPTRAP setup at the GSI in Darmstadt. The lowest yield handled to date was on the order of one particle per minute (delivered to the gas cell) in the case of ${ }^{256} \mathrm{Lr}$ corresponding to a cross section of about sixty nanobarn [18]. Recent upgrades to the SHIPTRAP setup have been implemented to boost its performance further in order to extend the reach to nuclides with much lower yield. First, a new cryogenic stopping cell has been installed. It features a larger stopping volume and a beam injection along the symmetry / extraction axis, which is in contrast to the previous gas cell where the beam was injected almost perpendicular. In addition, the operation at temperatures of about $40 \mathrm{~K}$ results in a higher cleanliness since potential impurities freeze out. In off-line measurements using a radioactive recoil ion source a extraction efficiency of up to $70 \%$ was determined [17]. For the integration of the cryogenic gas cell, the entire SHIPTRAP setup was recently relocated within the newly arranged experimental area behind the SHIP separator at the GSI. First online commissioning experiments with ${ }^{254}$ No resulted in an increased overall efficiency by at least a factor of five [26]. Further improvements are anticipated.

The second upgrade concerns the measurement technique employed to obtain the cyclotron frequency. Recently, the novel phase-imaging ion-cyclotron-resonance method technique has been developed at SHIPTRAP [27]. In this method the ion motion is imaged 
utilizing a spatially resolving ion detector, typically a microchannel plate detector with delay line anode. Choosing an appropriate ion optics with distortion free magnification allows to measure the phase of the (radial) ion motion from which the corresponding frequency can be obtained. The systematic uncertainties relevant to this method have been studied in depth [28]. Several mass measurements have shown that the novel method provides a forty times higher mass resolving power and a gain in precision by a factor of about five compared to the conventional scheme [27]. The new method has been applied to mass difference measurements of stable and long-lived nuclides, for example ${ }^{163} \mathrm{Ho}$ and ${ }^{48} \mathrm{Ca}$, reaching uncertainties of tens of electronvolts $[29,30]$. For the application to the heaviest elements in the context of nuclear structure studies uncertainties of a few $\mathrm{keV}$ suffice. Then, to reach the same uncertainties the method is about twenty-five times faster than the conventional method. This is beneficial for the access to shorter-lived nuclides. Furthermore, the high resolving power opens up new perspectives to study isomeric states with very low excitation energy, for example in ${ }^{255} \mathrm{Lr}$.

A key development to extend the reach to heavier nuclides with decreasing yield is the realization of single-ion mass measurement techniques. A technique that has already been developed for electrons, protons, and lighter stable ions, often highly charged, is the so-called Fourier-transform ion-cyclotron-resonance technique (FT-ICR) [31]. The method relies on a non-destructive image current detection. Trapped ions induce image charges into the trap electrodes due to their motion. Such weak currents on the order of 100 femtoamperes can be amplified by resonant tank circuits with high quality factor. For radioactive ions developments are presently underway, for example at TRIGA-TRAP [32], SHIPTRAP [33], and at the NSCL within the SIPT project [34].

In the future also time-of-flight mass spectrometers (ToF-MS) [35] are planned to be used for experiments on the heaviest nuclides. In recent years so-called multi-reflection (MR) ToF-MS have been developed. In these devices cooled short ion bunches are reflected between electrostatic mirrors. Thus, a compact setup can be maintained while providing high mass resolving powers up to about 200000 for millisecond flight times [36-38]. In contrast to conventional ToF-MS where the flight path limits the achievable precision to typically a level of $10^{-6}$ these devices can reach relative mass uncertainties of about $10^{-7}$ or even below [39]. MR-ToF-MS devices are thus suitable for mass measurements with a precision usually required to study global nuclear structure trends. These devices require low-energy bunched beams as in case of PTMS. Hence the beam preparation stages are usually the same involving a buffer gas stopping cell and an RFQ buncher. At RIKEN an MR-ToF-MS setup has been installed behind the new gas-filled recoil separator GARIS II [36]. First on-line measurements have been performed in neutron-deficient Fr isotopes [40].

All the different improvements will allow us to extend the reach of mass spectrometry to more exotic nuclides in the future to make a stronger push into the region of the heaviest elements.

\section{References}

[1] K. Blaum, Phys. Rep. 425, 1 (2006).

[2] H.J. Kluge, Int. J. Mass Spectrom. 349, 26 (2013).

[3] G. Bollen et al., Phys. Rev. Lett. 96, 152501 (2006).

[4] R. Van Dyck Jr. et al., Phys. Rev. Lett. 92, 220802 (2004).

[5] R.S. Van Dyck et al., Int. J. Mass Spectrom. 251, 231 (2006).

[6] L.S. Brown and G. Gabrielse, Rev. Mod. Phys. 58, 233 (1986).

[7] G. Bollen et al., J. Appl. Phys. 68, 4355 (1990). 
[8] M. Dworschak et al., Phys. Rev. C 81, 064312 (2010).

[9] J. Rissanen et al., Phys. Rev. C 83, 011301 (2011).

[10] L.-L. Andersson et al., GSI Scientific Report 2010, GSI Report 2011-1, p. 213.

[11] M. Block et al., Nature 463, 785 (2010).

[12] M. Block, Int. J. Mass Spectrom. 349, 94 (2013).

[13] M. Block, Nucl. Phys. A944, 471 (2015).

[14] J. Neumayr et al., Nucl. Instr. Meth. B244, 489 (2006).

[15] G. Savard, J. Phys.: Conf. Ser. 312, 052004 (2011).

[16] S. Eliseev et al., Nucl. Instr. Meth. B258, 479 (2007).

[17] C. Droese et al., Nucl. Instr. Meth. B338, 126 (2014).

[18] E.M. Ramirez et al., Science 337, 1207 (2012).

[19] M. König et al., Int. J. Mass Spectrom. 142, 95 (1995).

[20] L.L. Andersson et al., Nucl. Instr. Meth. A622, 164 (2010).

[21] D. Rudolph et al., GSI Scientific Report 2008, GSI Report 2009-1, p. 177.

[22] J.M. Gates, contribution to this issue (2016).

[23] M. Bender, W. Nazarewicz, P.G. Reinhard, Phys. Lett. B515, 42 (2001).

[24] N. Wang and M. Liu, J. Phys.: Conf. Ser. 420, 012057 (2013).

[25] M. Eibach et al., Phys. Rev. C 89, 064318 (2014).

[26] O. Kaleja et al., GSI Scientific Report, XXXX.

[27] S. Eliseev et al., Phys. Rev. Lett. 110, 082501 (2013).

[28] S. Eliseev et al., Appl. Phys. B 114, 107 (2014).

[29] S. Eliseev et al., Phys. Rev. Lett. 115, 062501 (2015).

[30] F. Köhler et al., Nature Communications 7,10246 (2016).

[31] A.G. Marshall, C.L. Hendrickson, G.S. Jackson, Mass Spectrom. Rev. 17, 1 (1998).

[32] J. Ketelaer et al., Eur. J. Phys. A 42, 311 (2009).

[33] C. Weber et al., Eur. Phys. J. A25, 65 (2005).

[34] R. Ringle, S. Schwarz, G. Bollen, Int. J. Mass Spectrom. 349, 87 (2013).

[35] H. Wollnik, Int. J. Mass Spectrom. 349, 38 (2013).

[36] Y. Ito et al., Nucl. Instr. Meth. B317, 544 (2013).

[37] W.R. Plaß, T. Dickel, C. Scheidenberger, Int. J. Mass Spectrom. 349, 134 (2013).

[38] R. Wolf et al., Nucl. Instr. Meth. A686, 82 (2012).

[39] P. Schury et al., Nucl. Instr. Meth. B335, 39 (2014).

[40] P. Schury et al., Nucl. Instr. Meth. B376, 425 (2016). 